\title{
La adquisición de competencias mediante la auto- nomía en el proceso de aprendizaje autorregulado
}

\author{
Sebastián SÁNCHEZ CASTILlO \\ Universitat de València \\ sebastian.sanchez@uv.es
}

Recibido: 04/07/2012

Aceptado: 22/10/2012

\begin{abstract}
Resumen
La autorregulación académica se ha establecido como una de las competencias básicas en el sistema educativo bajo el nuevo paradigma del Espacio Europeo de Enseñanza Superior. La autorregulación del aprendizaje no es una capacidad mental, como la inteligencia, o una habilidad, como la lectura, sino un proceso autodirigido a través del cual los aprendices transforman sus capacidades mentales en habilidades académicas. En la investigación que aquí se presenta, se consideran específicamente las dimensiones motivacionales (orientación intrínseca y extrínseca, valor otorgado a las tareas, locus de control para el aprendizaje, autoeficacia para el aprendizaje y ansiedad ante las evaluaciones) dirigido a estudiantes de Comunicación Audiovisual de la Universitat de València.
\end{abstract}

Palabras clave: Motivación; autorregulación académica; autoeficacia; resultados académicos.

\section{Competence Acquisition through Autonomy in the Self-regulated Learning Process}

\begin{abstract}
Academic self-regulation has become one of the basic competences in our educational system following the new paradigm of the European Space for Higher Education. Learning self-regulation is not a mental capacity such as intelligence or a skill such as reading but a self-directed process through which learners transform their mental capacities into academic skills. Motivational dimensions (e.g., intrinsic and extrinsic guidance, value given to activities, learning locus of control, learning self-efficiency and evaluation-related anxiety) are specifically considered in the present research focused on Media and Communication students at the University of Valencia.

Keywords: motivation, academic self-regulation, self-efficiency, academic performance.

\section{Referencia normalizada}

SÁNCHEZ CASTILLO, Sebastián (2012): "La adquisición de competencias mediante la autonomía en el proceso de aprendizaje autorregulado". Estudios sobre el mensaje periodístico. Vol. 18, núm. especial noviembre, págs.: 849-857. Madrid, Servicio de Publicaciones de la Universidad Complutense.
\end{abstract}

Sumario: 1. Introducción. 2. Metodología. 3. Resultados. 4. Discusión. 5. Referencias bibliográficas.

\section{Introducción}

En los estudios sobre la autorregulación del aprendizaje hay un interés creciente por explicar de qué manera los estudiantes acaban convirtiéndose en los directores de su proceso de aprendizaje (Zimmerman, 1989). La autorregulación académica se ha establecido como una de las competencias básicas en el sistema educativo bajo el nuevo paradigma del Espacio Europeo de Enseñanza Superior (EEES) y puede definirse como, el grado en que los estudiantes son participantes activos en sus propios procesos de aprendizaje desde un punto de vista metacognitivo, motivacional y comportamental (Zimmerman, 1989). 
Diversos autores afirman que las estrategias de aprendizaje son un constructo multidimensional, polisémico y confuso en ocasiones, del que se han dado múltiples definiciones (Gargallo, et al., 2009; Ayala, et al., 2004; Beltrán, 1993 y 2003; Bernad, 1999; Danserau, 1985; Kirby, 1984; Monereo, 1997; Monereo y Castelló, 1997; Nisbet y Shucksmith, 1987; Pozo, 1990; Weinstein y Danserau, 1985), si bien es cierto que en determinados momentos el énfasis a la hora de conceptualizarlas se puso en los aspectos.

La intervención por parte del estudiante sobre el ambiente y sobre las conductas desarrolladas por el propio estudiante hace referencia a cómo este es activo en su proceso de aprendizaje, de forma que una de las principales causas del fracaso de algunos estudiantes se sitúa en la incapacidad de gestionar dichos procesos y por tanto de autocontrolarse (Zimmerman, 1994). La generación de un sistema de control del proceso de aprendizaje ha sido asociada al término aprendizaje autorregulado.

Los profesores Suárez Riveiro, y Anaya Nieto (2004), indican que frente a la tradicional manera de entender el proceso de enseñanza/aprendizaje como un proceso unidireccional y pasivo por parte del alumno, se tiende a considerar una serie de elementos significativos que se encuentran en la mente del alumno y que comprenden tanto elementos cognitivos como elementos afectivos-motivacionales (p.e., sus atribuciones, conocimientos previos, autoconcepto, metas académicas, estrategias de aprendizaje, etc.). Se pasa así a considerar al alumno como agente activo y autodirigido (Beltrán, 1993), que construye, modifica y utiliza su conocimiento para interpretar situaciones en un determinado dominio y actuar dentro de él (Vermunt y Verloop, 1999).

La investigación ha venido centrándose en la gestión de los aspectos más conductuales y estratégicos, sin embargo, el aprendizaje autorregulado también hace referencia a la gestión de la propia motivación y afectividad. Es en este ámbito en donde se incluye la gestión de las orientaciones de meta, las cuales, a su vez, dirigen los aspectos más conductuales y cognitivos del aprendizaje. De esta forma, la gestión de las orientaciones de meta, es decir la activación y redirección de las razones del compromiso ante las tareas, permitirá a los estudiantes adaptarse a las demandas planteadas. Así, por ejemplo, un estudiante podría adoptar una orientación que prime el aprendizaje en sí mismo, en relación con un determinado tema o asignatura, y una orientación que busque únicamente el obtener buenas calificaciones, en relación con otro tema o asignatura. Dicho estudiante tendrá que gestionar su tiempo, esfuerzo, búsqueda de ayuda y estrategias cognitivas y metacognitivas de distinta forma en cada una de ellas. Pero también, dichas orientaciones de meta pueden necesitar ser redirigidas, sustituidas e incluso abandonadas en un determinado momento, dependiendo de las demandas contextuales y de sus propios intereses (Suárez, et al., 2001).

En el ámbito profesional se espera de los titulados que se incorporan al mismo, capacidad de utilizar información eficazmente para diferentes finalidades y de planificar el propio trabajo de forma independiente (Villardón, et al., 2007). La autonomía en el aprendizaje se entiende como la "facultad de tomar decisiones que permitan regular el propio aprendizaje para aproximarlo a una determinada meta, en el seno de unas condiciones específicas que forman el contexto de aprendizaje" (Monereo, 2001: 12). 
El desarrollo de la autonomía en el aprendizaje integrada con los correspondientes conocimientos y fortalecida con actitudes relevantes en el bien hacer científico y profesional, garantizan la adquisición de las competencias necesarias. Así queda expresado en el borrador de Real Decreto de 26 de junio de 2007, por el que se establece la ordenación de las enseñanzas universitarias oficiales, cuando se alude entre las competencias básicas que deben garantizar los estudios de Grado a "las habilidades de aprendizaje necesarias para emprender estudios posteriores con un alto grado de autonomía" junto a "aplicar conocimientos [...] de una forma profesional", "emitir juicios $[. .$.$] sobre temas relevantes de índole social, científica o ética"; y entre las que$ deben garantizar los estudios de Máster "las habilidades de aprendizaje que les permitan continuar estudiando de un modo que habrá de ser en gran medida autodirigido o autónomo" junto a "resolver problemas en entornos nuevos" o "comunicar [...] de un modo claro y sin ambigüedades".

Villardón, (et. al, 2007) señala que es importante que el curriculum universitario ayude a los estudiantes a convertirse en personas que gestionan sus propios recursos para aprender, creativas, preparadas para aprender a lo largo de la vida (Leinonen, et al., 2004).

En la investigación que aquí se presenta, se consideran específicamente las dimensiones motivacionales (orientación intrínseca y extrínseca, valor otorgado a las tareas, locus de control para el aprendizaje, autoeficacia para el aprendizaje y ansiedad ante las evaluaciones) evaluadas a través de las correspondientes subescalas del Motivated Strategies for Learning Questionnaire-MSLQ- (Pintrich, et al., 1993). Se trata de un método para evaluar la motivación de los estudiantes de Comunicación Audiovisual de la Universitat de València y conocer así la forma en que se puede desarrollar el citado proceso de autorregulación académico.

\section{Metodología}

El cuestionario MSLQ compuesto por 31 afirmaciones, fue presentado a estudiantes del Grado de Comunicación Audiovisual de la Facultad de Filología, Traducción y Comunicación en el campus de la Universidad de Valencia el 23 de mayo de 2012. Según la muestra de conveniencia (Igartua, 2006) y según los diferentes aspectos sociodemográficos considerados en total fueron encuestados 118 alumnos (M.edad=19,85 años, rango 18-46 años; 57,9\% mujeres, $\mathrm{n}=70$ ).

La recogida de datos se realizó en el aula de forma colectiva a alumnos previamente instruidos e informados del motivo del estudio. La participación fue voluntaria.

Los alumnos cursaban las asignaturas de $(n=53$, Teoría de la Imagen y Narrativa Audiovisual -TyN-, $2^{\circ}$ curso) y ( $\mathrm{n}=65$, Sociedad Actual- SA-, $1^{\circ}$ curso). De la totalidad de los alumnos, $(\mathrm{n}=101,83,5 \%)$ de los mismos, realizan los estudios a tiempo completo, y $(\mathrm{n}=17,14 \%)$ comparten los estudios con alguna actividad laboral. En cuanto a la nota media curricular de los alumnos en el momento de realizar el presente estudio, se extrae como aprobados $(n=12 ; 9,9 \%)$, notable $(n=82 ; 67,8 \%)$, y sobresaliente $(\mathrm{n}=24 ; 19,8 \%)$.

A cada una de los ítems que compone el cuestionario MSLQ se le ha dotado de un valor de 1 a 7, indicando al estudiante que si se identifica perfectamente con la ex- 
presión marque el 7; si no se ajusta en nada que marque el 1. El estudio tiene como objetivo conocer la capacidad de autogestión para el aprendizaje, y de estudiar algunos constructos estructurales y académicos.

El procedimiento analítico y los datos recabados por el cuestionario MSLQ (Pintrich, et al., 1991) se realizó "buscando técnicas de complementariedad" (Gargallo, et al., 2009: 427) mediante técnicas de correlación bivariada como Pearson $\left(r_{x y}\right)$ y contraste de hipótesis. Se ha empleado como software SPSS v.17.

\section{Resultados}

Tras analizar y concluir los datos estadísticos procedentes de las encuestas cumplimentadas por los alumnos, se ha comprobado en primer lugar que se detecta una media superior $(\mathrm{M}=5,30)$ en la dimensión de autoeficacia hacia el aprendizaje y la ejecución académica, (tabla 1). La puntuación menor se dirige a la dimensión que analiza la ansiedad ante las evaluaciones $(\mathrm{M}=4,43)$. En cuanto a la consistencia interna que justifica la fiabilidad, en la escala se han obtenido los siguientes datos según el coeficiente Alpha de Cronbach; escala (OI) $\alpha:, 59$; (OE) $\alpha:, 61 ;$ (VT) $\alpha:, 48$; (LC) $\alpha:, 57$; (AUT) $\alpha:, 52 ;$ (ANS) $\alpha:, 50$.

\section{${ }^{*}$ ) Media para los puntajes originales/no de ítems}

\begin{tabular}{llcc}
\hline Escala & $\mathrm{M}^{\left({ }^{*}\right)}$ & $\mathrm{Sd}$ & $\mathrm{Mdn}$ \\
\hline (1) Orientación Intrínseca (OI) & 5,20 & 1,42 & 5,50 \\
(2) Orientación Extrínseca (OE) & 5,02 & 1,67 & 5,25 \\
(3) Valor de la tarea (VT) & 4,97 & 1,54 & 5,33 \\
(4) Creencias sobre el lugar (LC) & 5,22 & 1,48 & 5,50 \\
(5) Percepción de autoeficacia (AUT) & 5,30 & 1,41 & 5,43 \\
(6) Ansiedad ante evaluaciones (ANS) & 4,43 & 1,88 & 6,00 \\
\hline
\end{tabular}

Tabla 1. Media; desviación estándar; mediana en MSLQ, $n=118$ Elaboración propia

A continuación se ha considerado el análisis de correlación bivariada de Pearson ${ }^{1}$, que oscila entre -1 y +1 . Ha de señalarse que la magnitud de la relación viene especificada por el valor numérico del coeficiente, reflejando el signo la dirección de tal valor. Así, tan fuerte es una relación de +1 como de -1 . En el primer caso la relación es perfecta positiva y en el segundo perfecta negativa. En sentido estricto, la correlación entre dos variables tan solo significa que ambas variables comparten información, que comparten variabilidad. Determinar el origen de la información, la fuente de la variabilidad -la causa- es una cuestión que no puede resolverse mediante recursos exclusivamente estadísticos. Por ello, mediante este análisis conoceremos la intensidad

${ }^{1}$ En el coeficiente de correlación de Pearson, pensado para variables cuantitativas (escala mínima de intervalo), es un índice que mide el grado de covariación entre distintas variables relacionadas linealmente. Puede haber variables fuertemente relacionadas, pero no de forma lineal; por ejemplo, la relación entre la ansiedad y el rendimiento tiene forma de U invertida. 
de la supuesta correlación, pero en ningún caso su causalidad, ya que correlación y causación no son términos equivalentes. Es necesario indicar que valores cercanos a 0 no significa que no exista asociación, sino como ausencia de correlación o asociación lineal.

Según se indica en la tabla 2, se descubre una gran correlación entre el ítem 9 "si no aprendo los contenidos en esta materia será responsabilidad mía" correspondiente a las creencias sobre lugar de control (LC), locus y sensación de control y la variable "sexo", $(\mathrm{r}[118]=-, 282, \mathrm{p}<, 002)$. También se detecta una gran asociación entre la variable o ítem 19"cuando hago un examen me siento intranquilo" que evalúa los componen cognitivos y emocionales de la ansiedad ante las evaluaciones académicas, $(\mathrm{r}[118]=-, 237, \mathrm{p}<, 010)$. Por último el ítem n $\mathrm{n}^{\circ} 25$ "si no entiendo los contenidos de esta materia, será porque no me esfuerzo lo suficiente" que alude a la dimensión que avalúa el grado en que el sujeto percibe que su esfuerzo se traducirá en resultados positivos, también presenta una alta asociación a la variable "sexo", $(r[118]=-, 242$, $\mathrm{p}<, 008)$.

En cuanto a la variable "edad" y aunque esta es muy similar en los encuestados, los resultados obtenidos no carece de interés. Se descubre una asociación lineal intensa entre esta variable y el ítem 17 "Estoy muy interesado en los contenidos de esta materia" sobre el valor de la tarea y evalúa el grado de importancia, utilidad e interés que el estudiante concede a las tareas académicas, $(\mathrm{r}[118]=, 184 \mathrm{p}<, 047)$. De la misma forma "pienso que me resulta útil aprender los contenidos de esta materia", $(\mathrm{r}[118]=, 183 \mathrm{p}<, 048)$ y "en esta materia prefiero escoger tareas y actividades con las que pueda aprender incluso cuando no me garanticen una buena nota", $(r[118]=, 219$ $\mathrm{p}<, 017)$. Finalmente el ítem "comprender los contenidos de esta materia es muy importante a mí" muestra también una ligera correlación con la nota media obtenida en el grado analizado, $(r[118]=, 206 \mathrm{p}<, 025)$.

Respecto a la variable "nota", se descubre una asociación entre esta y el ítem "en esta materia, prefiero contenidos que realmente me desafíen a aprender cosas nuevas" de la orientación intrínseca, $(\mathrm{r}[118]=-, 215 \mathrm{p}<, 020)$, y con el ítem "pienso que podré utilizar lo que aprendo en esta materia en otras asignaturas" en la dimensión de creencias sobre lugar de control y sensación de control, (r[118]=,193 p<,036).

La dedicación a los estudios total o compartida con otra actividad también está asociada al ítem "si estudio de la forma adecuada, podré aprender los contenidos de esta materia" dimensión que evalúa el grado en que el sujeto percibe que su esfuerzo se traducirá en resultados positivos $(\mathrm{r}[118]=-, 286 \mathrm{p}<, 002)$. También el ítem "pienso que me resulta útil aprender los contenidos de esta materia" en la dimisión del valor de la tarea $(\mathrm{r}[118]=-, 211 \mathrm{p}<, 022)$, y "me gusta esta materia" en la misma dimensión $(r[118]=-, 290 \mathrm{p}<, 001)$, en este caso con una correlación mucho más intensa. 


\begin{tabular}{|c|c|c|c|c|}
\hline ítem/MSQL & Sexo & Edad & Nota & Dedicación \\
\hline $\begin{array}{l}\text { 1. En esta materia, prefiero contenidos que } \\
\text { realmente me desafien a aprender cosas nuevas }\end{array}$ & 088 & 145 &,$- 215^{*}$ &,- 113 \\
\hline $\begin{array}{l}\text { 2. Si estudio de la forma adecuada, podré aprender } \\
\text { los contenidos de este materia }\end{array}$ &,- 019 & 108 &,- 090 &,$- 286^{* *}$ \\
\hline $\begin{array}{l}\text { 4. Pienso que podré utilizar lo que aprendo en esta } \\
\text { materia en otras asignaturas }\end{array}$ &,- 168 & 125 & ,193* &,- 172 \\
\hline $\begin{array}{l}\text { 9. Si no aprendo los contenidos en este materia será } \\
\text { responsabilidad mía }\end{array}$ &,$- 282^{* *}$ & ,001 &,- 093 &,- 117 \\
\hline $\begin{array}{l}\text { 17.Estoy muy interesado en los contenidos de este } \\
\text { materia }\end{array}$ &,- 098 &, $184^{*}$ &,- 095 &,- 016 \\
\hline 19. Cuando hago un examen me siento intranquilo &,$- 237^{* *}$ & 127 &,- 054 & 155 \\
\hline $\begin{array}{l}\text { 22. Lo que más me satisface en esta materia es } \\
\text { tratar de entender los contenidos a fondo }\end{array}$ &,$- 216^{*}$ & 090 & ,037 &,- 066 \\
\hline $\begin{array}{l}\text { 23.Pienso que me resulta útil aprender los } \\
\text { contenidos de esta materia }\end{array}$ &,- 139 &, $183^{*}$ & ,048 &,$- 211^{*}$ \\
\hline $\begin{array}{l}\text { 24. En esta materia prefiero escoger tareas y } \\
\text { actividades con las que pueda aprender incluso } \\
\text { cuando no me garanticen una buena nota }\end{array}$ &,- 094 &, $219^{*}$ &,- 008 & 105 \\
\hline $\begin{array}{l}\text { 25. Si no entiendo los contenidos de esta materia, } \\
\text { será porque no me esfuerzo lo suficiente }\end{array}$ &,$- 242^{* *}$ & ,033 & 112 &,- 153 \\
\hline 26. Me gusta esta materia &,- 154 & 118 &,- 131 &,$- 290 * *$ \\
\hline $\begin{array}{l}\text { 27. Comprender los contenidos de esta materia es } \\
\text { muy importante a mí }\end{array}$ &,- 087 & ,206* &,- 083 &,- 131 \\
\hline
\end{tabular}

Tabla 2. Coeficiente de Correlación de Pearson ( $r$ )

Elaboración propia

\section{Discusión}

La sociedad del conocimiento exige a la universidad una formación profesionalizada y la formación de ciudadanos capaces de construir sus propias competencias y conocimiento para el ejercicio de sus funciones. En el ámbito profesional se espera capacidad de manejar información y de planificar el propio trabajo de forma independiente. Para ello, se requieren ciertas capacidades de carácter holístico. Es importante que el curriculum universitario ayude a los estudiantes a convertirse en personas que gestionan sus propios recursos para aprender, creativas, preparadas para aprender a lo largo de la vida (Villardón, et al., 2007).

A partir de las competencias capaces de favorecer la autonomía de los alumnos en el aprendizaje enumeradas por Monereo y Pozo (2003), pensar, cooperar, comunicar, empatizar, ser crítico y automotivarse, con los datos obtenidos en esta investigación se han obtenido varias conclusiones.

En primer lugar se evidencia que los estudiantes de Comunicación Audiovisual de la Universitat de València encuestados conceden un mayor valor a la dimensión de au- 
toeficacia hacia el aprendizaje y la ejecución académica, mostrando una alta confianza y seguridad en sus habilidades para desarrollar las tareas y actividades planteadas, donde el estudiante percibe que obtendrá resultados (nivel de ejecución) satisfactorios en la tarea.

También se manifiesta que los estudiantes perciben de forma muy notable que su esfuerzo se traducirá en resultados positivos; es decir que los resultados académicos son producto de su esfuerzo personal y de factores externos como el profesor. Esta dimensión favorece la creencia que el sujeto descubre que puede controlar el nivel de logro, y que puede actuar cuando sea necesario para mejorar los resultados.

Por otra parte, se establece una relación significativa en especial el perfil de dedicación a los estudios y la nota media obtenida, con las creencias sobre lugar de control (LC), locus y sensación de control que evalúa el grado en que el sujeto percibe que su esfuerzo se traducirá en resultados positivos. También se descubre que los estudiantes de Comunicación Audiovisual de la Universitat de València, según su sexo, edad y dedicación muestran un gran interés por evaluar las tareas académicas y por lo tanto una gran implicación por el aprendizaje.

Con los datos obtenidos es posible establecer unas consideraciones específicas respecto a estos alumnos. Se ha detectado que los estudiantes de esta área de conocimiento muestran muy poca importancia, utilidad o interés a las tareas académicas, resultando mínima la implicación con el aprendizaje, aunque como se ha afirmado anteriormente muestra gran confianza y seguridad ante estas tareas.

En cuanto a la ansiedad que muestran los estudiantes ante los exámenes, los resultados se sitúan por debajo de la media. Teniendo en cuenta que esta dimensión tiene una correlación negativa, los alumnos no muestran un especial impacto emocional frente a las evaluaciones.

Los resultados muestran un conjunto de datos muy útiles para realizar una revisión de los procesos instruccionales, mecanismos de evaluación del rendimiento y los procedimientos de evaluación psicológica en los alumnos de esta área de conocimiento.

\section{Referencias bibliográficas}

AYALA, Carlos; MARTÍNEZ, Rosario y YUSTE, Carlos (2004): CEAM. Cuestionario de estrategias de aprendizaje y motivación. Barcelona, Instituto de Orientación Psicológica EOS.

BELTRÁN, Jesús (2003):” Estrategias de aprendizaje”. Revista de Educación, n 332 , pp. 55-73.

BELTRÁN, Jesús (1993): Procesos, estrategias y técnicas de aprendizaje. Madrid, Síntesis/ Psicología.

BERNAD, Juan Antonio (1999): Estrategias de aprendizaje. Madrid, Bruño.

DANSERAU, Donald (1985): "Learning Strategy Research". En H.F. O’Neil (Ed.), Learning Strategies, pp. 209-240. Nueva York, Academic Press.

GARGALLO, Bernardo; SUÁREZ-RODRIGUEZ, Jesús y PÉREZ-PÉREZ, Cruz (2009): "El cuestionario CEVEAPEU. Un instrumento para la evaluación de las es- 
trategias de aprendizaje de los estudiantes universitarios". Relieve, v. 15, $\mathrm{n}^{\mathrm{0}} 2$, pp. $1-31$.

GARGALLO LÓPEZ, Bernardo; SUÁREZ-RODRÍGUEZ, Jesús y FERRERAS REMESAL, Alicia (2007): "Estrategias de aprendizaje y rendimiento académico en estudiantes universitarios". Revista de Investigación Educativa, v. 25, nº 2, pp. 421-441.

IGARTUA PEROSANZ, Juan José (2006): Métodos cuantitativos de investigación en comunicación. Barcelona, Editorial Bosch.

KIRBY, John (1984): Cognitive strategies and educational performance. Orlando, Academic Press.

LEINONEN, Niina; PARTANEN, Johannes; PALVIAINEN, Petri y GATES, Marietta (2004): The Team Academy. A true story of a Community that Learns by doing. Juva, PS-kustannus.

MONEREO, Carles (1997): "La construcción del conocimiento estratégico en el aula". En Mª.L. Pérez Cabaní, La enseñanza y el aprendizaje de estrategias desde el currículo. Gerona, Horsori, pp. 21-34

MONEREO, Carles (2001): "La enseñanza estratégica, enseñar para la autonomía". En C. Monereo (coord..): Ser estratégico y autónomo aprendiendo. Unidades didácticas de enseñanza estratégica. Barcelona, Grao.

MONEREO, Carles y CASTELLÓ, Montserrat (1997): Las estrategias de aprendizaje. Cómo incorporarlas a la práctica educativa. Barcelona, Edebé.

MONEREO, Carles y POZO, Juan Ignacio (2003): La universidad anta la nueva cultura educativa. Madrid, Síntesis.

NISBET, John y SHUCKSMITH, Janet (1987): Estrategias de aprendizaje. Madrid, Santillana.

PINTRICH, Paul; SMITH, David; GARCÍA, Teresa y McKEACHIE, Wilbert (1993). "Reliability and predictive validity of the Motivated Strategies for Learning Questionnaire (MSLQ)". Educational and Psychological Measurement, $\mathrm{n}^{\circ}$ 53, pp. 801813.

PINTRICH, Paul; SMITH, David; GARCÍA, Teresa y McKEACHIE, Wilbert (1991): A manual for the use of the Motivated Strategiesfor Learning Questionnaire (MSLQ). NCRIPTAL-91-B-004.Ann Arbor, MI.

POZO Juan Ignacio (1990): "Estrategias de aprendizaje". En C. COLL, J. PALACIOS y A. MARCHESI: Desarrollo psicológico y educación, II. Psicología de la educación. Madrid, Alianza, pp. 199-221.

SUÁREZ RIVEIRO, José Manuel; GONZÁLEZ CABANACH, Ramón; ABALDE PAZ, Eduardo y VALLE ARIAS, Antonio (2001): "Un modelo explicativo de las influencias de las orientaciones de meta sobre la autorregulación del aprendizaje". Revista de Investigación Educativa, vol. 19, nº 1, pp. 249-262. 
SUÁREZ RIVEIRO, José Manuel y ANAYA NIETO, Daniel (2004): “Educación a distancia y presencial: diferencias en los componentes cognitivo y motivacional de estudiantes universitarios". Revista Iberoamericana de Educación a Distancia RIED, vol 7, pp. 65-75.

VERMUNT, Jan y VERLOOP, Nico (1999): "Congruence and friction between learning and teaching”. Learning and Instruction, $\mathrm{n}^{\circ}$ 9, pp. 257-280.

VILLARDÓN GALLEGO, Lourdes; ELEXPURU ALBIZURI, Itziar y YÁNIZ, Concepción (2007): “Autonomía, condición indispensable de la competencia para aprender Datos preliminares de un estudio". Congreso Internacional RED-U 2-07. El desarrollo de la autonomía en el aprendizaje. Universitat Pompeu Fabra. Barcelona, 5 y 6 de Julio.

WEINSTEIN, Claire y DANSERAU, Donald (1985): "Learning strategies: the how of learning". En J.W. Segal et al., Thinking and learning strategies, Hillsdale: Erlbaum, pp. 125-142.

ZIMMERMAN, Barry (1989): “A social-cognitive view of self- regulated academic learning”. Journal of Educational Psychology, $\mathrm{n}^{\circ}$ 81, pp. 329-339.

ZIMMERMAN, Barry (1994): "Dimensions of academic self-regulation: A conceptual framework for education". En D.H. Schunk y B. J. Zimmerman (Eds.), Selfregulation of learning and performance: Issues and educational applications. Hillsdale, New Jersey, Lawrence Erlbaum Associates.

\section{Sebastián SÁNCHEZ CASTILLO}

sebastian.sanchez@uv.es

Universitat de València

Facultat de Filologia, Traducció i Comunicació.

Departament de Teoria dels Llenguatges i Ciències de la Comunicació.

Avd. Blasco Ibáñez, n 32, 4610 (Valencia) 